\title{
Genèse des crues normales dans les petits bassins versants ruraux
}

\author{
Normal runoff generation in small agricultural catchments
}

\author{
par C. Gascuel-Odoux, P. Merot, P. Durand et J. Molenat \\ Unité Sol et Agronomie de Rennes-Quimper, INRA
}

The small catchments with a Strahler order under 3 are adequate to identify the main processes of storm flow generation. The paper focused on the results obtained on intensive agricultural catchments located in Western France. The endmember mixing analysis (EMMA), applied to two high frequency storm events showed that stormflow is due to different hydrological endmembers, whose contribution varies strongly both within storm event and from one event to another. The processes are highly non-linear. Three points are detailed : the delineation of the contributive area; the importance and variability of surface runoff; the dynamics of groundwater on the hillslope as linked to runoff generation.

- The studies on the delineation of the saturated bottom lands, considered as the runoff contributive areas, to better constrain hydrological models such as TopModel showed: $i$ ) that the location of these area is related to the elevation relative to the river level; a modification of the slope computation in the Kirby index is proposed; ii) the increase of the connectivity of the saturated areas in wet periods; iii) the interest of radar data to better constrain TopModel.

- The surface runoff is not very high but does vary depending on soil surface and hydric conditions along the hillslope and during the year. Experimental and numerical simulations showed the effect of the rugosity at different scales on runoff generation.

The analysis of the seasonal and within-event responses of the water table confirmed the predominant role of the groundwater in storm flow generation in this physical context.

Finally, this paper shows how the structure of the landscape, including the different linear networks, can modify the storm flow generation in the physiographic context of Western France. As an example, the network of hedgerows strongly modifies the potential length of surface runoff and the dynamics of the groundwater close to the contributive area.

\section{I INTRODUCTION}

Les crues de grandes fréquences ont fait l'objet de nombreux travaux, en particulier en zones agricoles intensives, afin de mieux connaître le fonctionnement hydrologique et hydrochimique des bassins versants. Dans ces situations, il s'agit de mieux connaître le cheminement de l'eau vecteur, pour in fine mieux comprendre l'origine des pollutions agricoles diffuses. Nous nous focaliserons dans cette présentation sur les travaux spécifiques menés sur les crues et l'origine des écoulements rapides, dans l'Ouest de la France, sur des petits bassins versants ruraux en zone d'agriculture intensive.

Partant d'une vision globale des écoulements à l'exutoire d'un bassin, nous montrerons dans un premier temps que les hydrogrammes de crues normales sont la résultante, à l'opposé des crues extrêmes, de la mobilisation de l'ensemble des réservoirs hydrologiques du bassin versant. Remontant aux processus, nous présenterons dans un deuxième temps des développements basés sur le concept de source à surface variable. Dans un troisième temps, nous détaillerons des approches très récentes sur le ruissellement de versant et la dynamique de la nappe. Enfin, l'influence sur l'hydrologie de la structuration du paysage par le réseau bocager sera évoquée.

\section{II — L'HYDROGRAMME D'UNE CRUE NORMALE : LA MOBILISATION DE L'ENSEMBLE DES RÉSERVOIRS DU BASSIN VERSANT}

Les modèles de mélange basés sur la confrontation de la composition chimique ou isotopique des eaux de la rivière à celle des différents réservoirs hydrologiques du bassin sont une méthode particulièrement adaptée aux petits bassins, où cette identification des différents réservoirs est possible. Ainsi, un premier travail [1] avait montré, dans un bassin versant d'une dizaine d'ha des environs de Rennes, que $12 \%$ au plus de l'hydrogramme de crue provenait de la pluie, le 


\begin{tabular}{|l|l|l|l|l|}
\hline & $\begin{array}{l}\text { Nappe } \\
\%\end{array}$ & $\begin{array}{l}\text { Pluie } \\
\%\end{array}$ & $\begin{array}{l}\text { Zone humide } \\
\%\end{array}$ & $\begin{array}{l}\text { Versant } \\
\%\end{array}$ \\
\hline $1^{\text {ere }}$ crue & 31,3 & 13,2 & 34,2 & 20,7 \\
\hline $2^{\text {ième }}$ crue & 19,2 & 26,7 & 34,2 & 19,9 \\
\hline
\end{tabular}

Tableau 1 : Contribution des 4 pôles à l'écoulement pour 2 événements [3].

gonflement de l'hydrogramme lors de la crue étant principalement généré par de l'eau provenant à la fois de la nappe et des horizons superficiels du sol. Cette approche a été reprise plus récemment sur le bassin versant de recherche du CoëtDan, soit à partir d'un modèle de mélange à 4 composantes [2], soit à partir du formalisme de la méthode EMMA - End Member Mixing Analysis - |3|. Les crues étudiées ont été suivies à l'exutoire d'un sous-bassin amont du B.V. du CoëtDan, le bassin de Kervidy de $3 \mathrm{~km}^{2}$ de superficie. Les conditions d'application des méthodes de mélange ont été développées par Durand et al. [3] : le choix des pôles de mélange est déduit de la connaissance $a$ priori que l'on a du bassin versant et de l'identification des différents réservoirs susceptibles de contribuer au mélange. Quatre pôles ont ainsi été définis à partir des études pédologiques et hydrodynamiques réalisées sur ce bassin versant $[4]$ : les sols du bassin versant ont été découpés selon deux domaines, les sols du versant et ceux de la zone riveraine ; l'écoulement de base correspondant à la nappe est le troisième pôle ; enfin, le ruissellement de surface correspondant à la pluie est le dernier pôle. Chacun de ces pôles possède une signature chimique ou isotopique particulière. Le choix des traceurs, nécessairement trois traceurs indépendants pour quatre pôles, s'est porté sur les nitrates, les chlorures et le sodium ou l'oxygène 18 , qui présentaient entre chaque pôle des différences significatives. Les autres éléments chimiques analysés ont été utilisés dans la phase de validation. D'autres traceurs de moindre interaction avec l'agriculture ont été récemment explorés sur ce bassin versant en collaboration avec l'université de Rennes [5].

Les résultats (figure 1) montrent que les hypothèses posées sont raisonnables : la plupart des variations chimiques sont

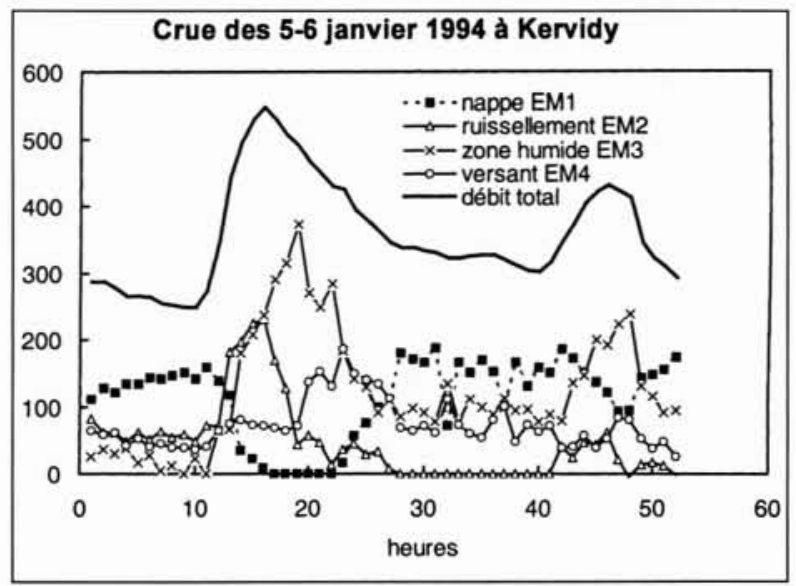

1. Débit total et variations des différentes composantes hydrologiques au cours d'une crue à partir de la méthode de la décomposition chimique des hydrogrammes de crue. expliquées par le modèle, et la décomposition de l'hydrogramme donne une image cohérente de l'origine de l'eau dans le bassin versant lors de la crue. On constate notamment (tableau 1) :

- la nécessité de faire intervenir effectivement les quatre réservoirs pour expliquer l'ensemble des variations observées ;

- une contribution moyenne, assez variable cependant selon les événements, mais toujours significative des différents réservoirs ;

- la diminution systématique, si ce n'est la disparition de la contribution de la nappe, lors de la montée de l'hydrogramme ;

- la contribution dominante de la zone humide ;

- une contribution de l'eau des versants, sans mélange dans la zone humide, grâce à l'existence de courts-circuits (macropores, drainage agricole, fossés).

Ces conclusions nous ont conduits à approfondir certains de ces écoulements : le fonctionnement de la zone saturée contributive, le ruissellement de surface et l'écoulement de nappe.

\section{III - LE FONCTIONNEMENT DES ZONES CONTRIBUTIVES}

Si le concept de zone contributive a constitué une avancée majeure en termes de modélisation hydrologique, parfois irritant par son ubiquité, sa validation sur le terrain et la définition de son domaine d'application ont été beaucoup plus progressives, du fait de la difficulté de cartographier les zones saturées souvent hautement dynamiques. Trois questions ont été abordées, qui toutes trois tentent de valider et de préciser la notion de zone contributive : i) quels sont les facteurs spatiaux de contrôle de l'humidité au champ ? ii) quelle est la dynamique spatiale des zones saturées ? iii) enfin comment mesurer ou approcher sur le terrain les zones saturées, notamment à l'aide de la télédétection ? L'ensemble de ces questions ont été abordées sur le bassin du Coët-Dan, à l'aide de nombreuses campagnes de mesures de l'humidité basées sur des semis denses de prélèvements ou de mesures TDR, par cartographie des limites de zones saturées, ou par télédétection radar satellitale.

\subsection{Les facteurs de contrôle de la distribution spatiale de l'humidité}

Les mesures de terrain faites hors période de crue (figure 2) ont mis en avant un contrôle de la distribution spatiale de l'humidité par les conditions topographiques aval, c'est-à-dire relatives au cours d'eau. On propose [6] ainsi un premier indice, qui est la dénivelée entre un point donné et le réseau hydrographique, calculé en tenant compte de l'arbre de drainage. Dans le domaine du versant, la teneur en eau moyenne 


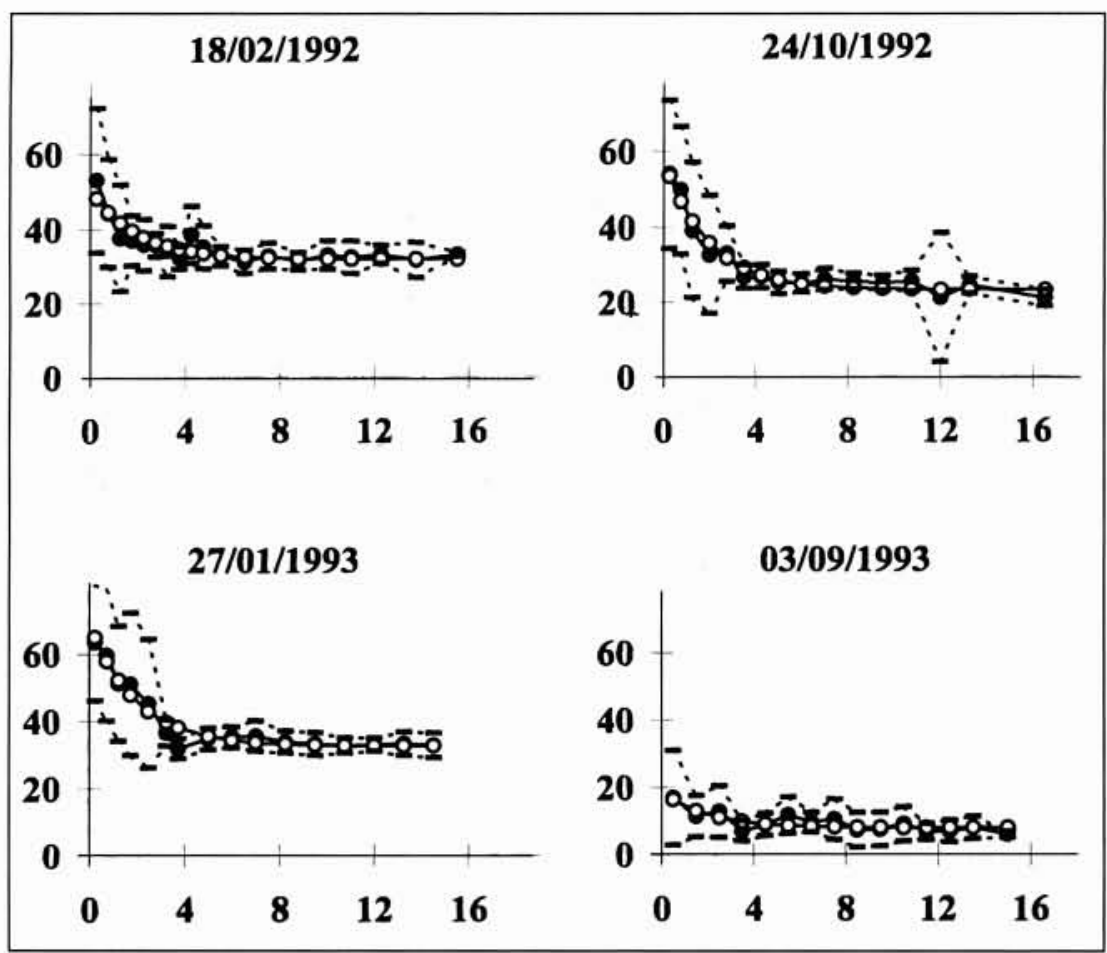

2. Moyenne et écart-type de la teneur en eau pondérale en \% en fonction de la dénivelée par rapport au ruisseau en mètres à différentes dates sur le bassin versant de Kervidy [5]. dité de cette approche de modélisation. Les prédictions de la superficie du domaine saturé en fonction des chroniques climatiques sont relativement bonnes, que ce soit à l'échelle de la crue ou à l'échelle de quelques mois [9]. A l'opposé, ce modèle ne prédit pas bien la localisation et la forme de ces surfaces, ni leurs dynamiques temporelles. Les zones saturées sont constituées d'un patchwork de petits domaines déconnectés les uns des autres pour les faibles niveaux de saturation ; lorsque la saturation du bassin s'étend, ces petits domaines se connectent et s'accroissent selon de nombreuses digitations. Dans un cas comme dans l'autre, cette localisation dépend d'une organisation topographique, pédologique ou anthropique observée à l'échelle de quelques mètres. Celle-ci ne peut être prise en compte dans un modèle fonctionnant sur une maille topographique trop large. Elle est cependant très importante car elle conditionne la connectivité des écoulements dans ce domaine de fond de vallée. ne varie pas avec la dénivelée et présente une faible variabilité spatiale ; à l'opposé, à l'aval, elle décroît exponentiellement en fonction de la dénivelée par rapport au ruisseau et présente une forte variabilité spatiale. Un seuil topographique, ici de $4 \mathrm{~m}$, constant semble-t-il en fonction du temps, délimite les 2 domaines, celui du versant et celui du bas fond. Cette limite correspond approximativement à une délimitation moyenne du domaine concave de bas de versant.

Alors que dans l'indice topographique défini par Kirkby c'est la pente locale qui intervient, nous avons proposé d'introduire dans cet indice une pente globale, calculée sur la partie du versant entre le point considéré et le réseau hydrographique, créant ainsi un indice topographique de Kirkby "aval". Outre le gain en robustesse de cet indice qui lisse ainsi les imperfections du MNT, cette adaptation limite le rôle prééminent donné à l'aire drainée amont des versants et rend mieux compte d'un drainage de la nappe contrôlé par des gradients de pente globaux.

Ces deux indices sont complémentaires : le second est déterminant pour estimer la distribution spatiale des déficits de saturation, en relation avec la dynamique de la nappe (périodes de crues); le premier correspond à une moyenne spatiale relative à une extension haute de la nappe (périodes hors crues). Le caractère opérationnel de ces deux indices a été largement reconnu depuis dans le contexte armoricain, notamment pour la prédiction des sols hydromorphes [7].

\subsection{La modélisation de la dynamique spatiale des zones saturée}

La confrontation [8] de mesures de l'extension des zones saturées à des prédictions par modélisation hydrologique par TopModel (figure 3 ) permet de préciser le domaine de vali-
3.3 La localisation de l'extension des zones saturées à l'aide de la télédétection

La télédétection radar, de par ses propriétés, constitue $a$ priori un outil particulièrement intéressant pour mesurer la dynamique des zones saturées contributives [10]. Si la mesure effective des zones humides et plus généralement de l'humidité des sols avec les capteurs actuels est encore inaccessible de façon opérationnelle, le bilan de l'utilisation du radar appliqué à la détection des zones saturées est loin d'être négatif.

- le suivi multi-temporel (à 3 jours d'intervalle) a permis de révéler la structure spatiale de la dynamique de ressuyage des sols, à l'échelle de la totalité d'un bassin versant de $12 \mathrm{~km}^{2}$. Il a permis de calculer un indice de saturation potentielle, basé sur la persistance de l'humidité, indice déduit des données radar et qui caractérise les zones les plus propices à se saturer [11].

- le couplage de cet indice avec l'indice topographique de Kirkby permet d'améliorer les prédictions spatialisées liées au seul indice topographique,

- enfin, cet indice a été appliqué à la modélisation hydrologique pour contraindre et réduire fortement l'espace des paramètres tel qu'il apparaît lors de l'application de la méthodologie GLUE, développée par Beven [12].

L'ensemble de ces travaux confirme la pertinence du concept de zone contributive notamment sur zone de socle, sous climat tempéré humide, et l'intérêt du formalisme développé initialement par Beven et Kirkby, enrichi ici par de nouveaux apports. Bien que cela n'ait pas pu être développé ici, il faut également souligner la convergence de ces travaux avec ceux menés par les pédologues sur les sols hydromorphes et par les géochimistes sur les zones riveraines anoxiques, qui font des zones contributives un élément clé du fonctionnement hydrogéochimique des paysages. 


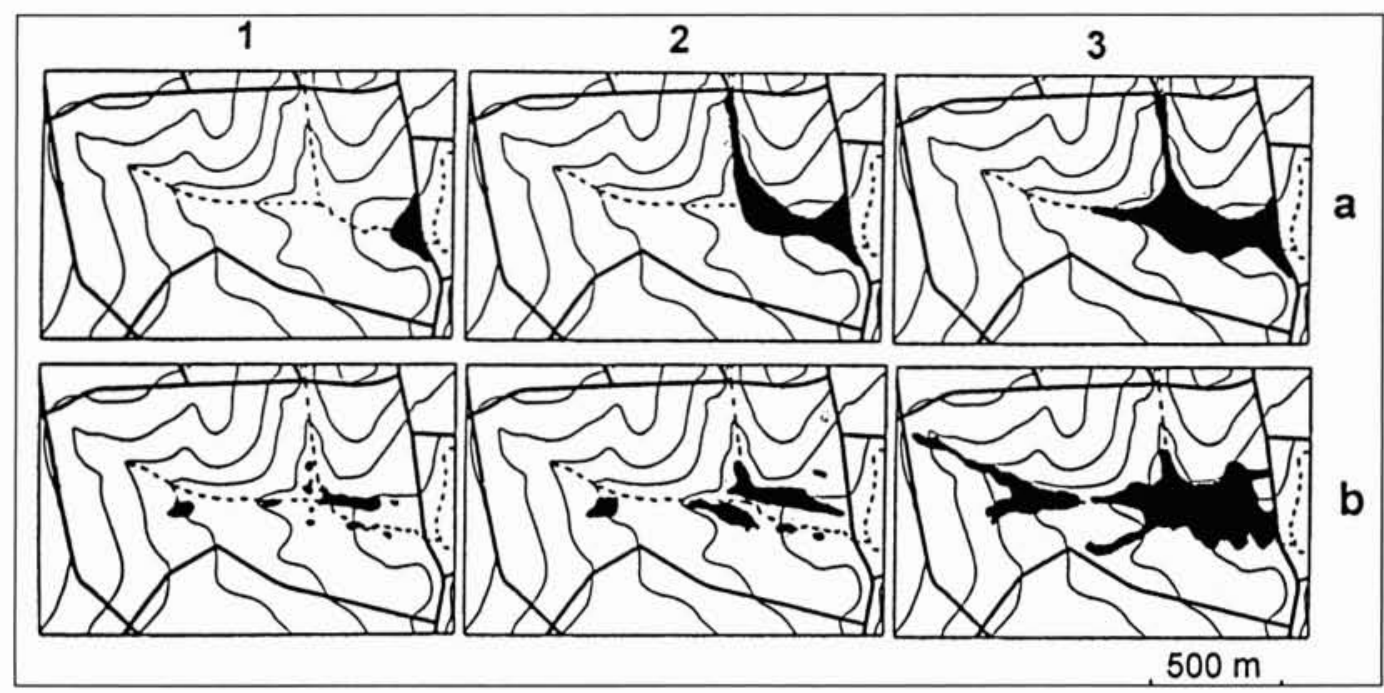

3. Extension des zones saturées à 3 dates de saturation : approche par modélisation (en haut), et par mesures de terrain (en bas) [7].

\section{IV — LA PROPAGATION DU RUISSELLE- MENT DE SURFACE LE LONG DU VERSANT}

Si les processus majeurs conditionnant la dynamique hydrologique en zone de socle et sous climat tempéré humide sont liés à la présence d'une zone humide de fond de vallée, le ruissellement de surface présent sur les versants est important pour au moins deux raisons : d'une part la forte dégradation des couches de surface par l'activité agricole en zone d'agriculture intensive facilement observable sous les passages de roues ou dans les cultures peu couvrantes, peut induire une forte diminution de l'infiltrabilité des sols : d'autre part le ruissellement est le vecteur privilégié de certains polluants - produits phytosanitaires, phosphore - qui affecte de manière préoccupante la qualité de l'eau. Dans un premier temps, nous rapporterons les conclusions du suivi du ruissellement de versant par un dispositif comprenant de nombreux collecteurs. Dans un deuxième temps, des simulations tant expérimentales que numériques démontreront le rôle important de la rugosité de surface selon différentes échelles spatiales sur la propagation du ruissellement.

\subsection{Distribution spatiale et temporelle du ruisselle- ment de versant et contrôles}

Un réseau de collecteurs des eaux de ruissellement répartis sur un versant d'un hectare a mis en évidence les différents paramètres contrôlant le ruissellement [13]. Ce travail a été mené sur une portion de versant correspondant à une seule parcelle agricole, homogénéisant ainsi les conditions de surface. La parcelle, de relief modéré, de forme convexoconcave, était cultivée en monoculture de maïs ensilage, le sol restant sans couverture végétale durant l'hiver. Le semis étant réalisé dans le sens de la pente, le système géométrique

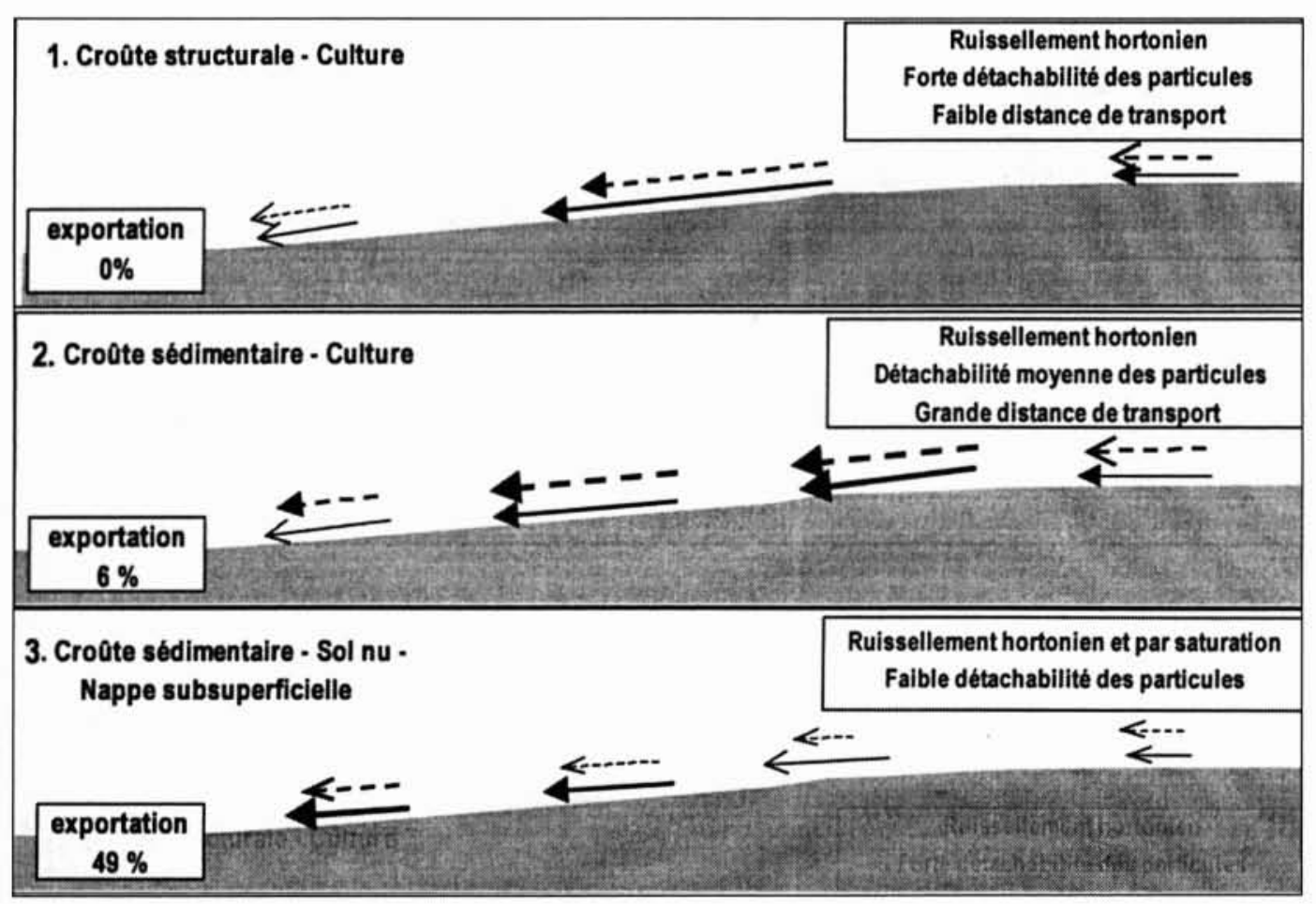

4. Schéma synthétique de la distribution spatiale du ruissellement et des transports solides dans le versant, à l'échelle d'une année culturale [20]. 
d'écoulement est simple, canalisé selon une même direction, par la pente et l'inter-rang de culture.

Trois périodes, selon le type d'averse, l'état hydrique du versant et les états de surface du sol, et deux domaines, délimités par la rupture de pente, ont été définis, à partir de l'occurrence et de l'intensité du ruissellement et de l'érosion, mesuré globalement averse après averse au cours de l'année (figure 4). Au printemps, période d'établissement d'une croûte de battance, le ruissellement porte sur de courtes distances, mais présente de fortes concentrations en charge solide. La variabilité spatiale est faible. Durant la période estivale le ruissellement a lieu préférentiellement dans le domaine de plus grande pente, mais est très fréquemment stoppé à la rupture de pente de bas de versant, excepté pour les averses les plus importantes. C'est du ruissellement hortonien. Les charges solides sont élevées. Durant cette période, la variabilité spatiale pour un même niveau de pente est forte. A l'opposé, durant l'hiver, le ruissellement a lieu préférentiellement en bas de versant, du fait de la saturation rapide des sols, et présente une faible variabilité spatiale. C'est du ruissellement par saturation, qui peut, selon l'état hydrique du bassin versant, remonter assez haut dans le versant.

Les trois périodes correspondent ainsi à des contrôles différents du ruissellement : 1) contrôle lié à des processus de dégradation de surface lors de la première période ; 2) contrôle par la topographie lors de la seconde période, mais avec une forte variabilité spatiale et temporelle ; 3) contrôle par l'hydrologie du versant lors de la troisième période.

On observe par ailleurs une évolution temporelle des propriétés hydrodynamiques des couches de surface nettement différenciées en fonction de la topographie. Ainsi, la conductivité hydraulique, homogène sur le versant en début de culture, diminue fortement au cours de l'année. Cette diminution au départ plus rapide à l'amont qu'à l'aval, du fait des variations spatiales de matières organiques dans le versant, est, en fin de saison hivernale, plus faible d'un facteur log à l'amont qu'à l'aval, du fait des variations spatiales de l'humidité dans le versant [14].

\subsection{Le rôle spécifique de la rugosité dans la propagation du ruissellement}

La rugosité du sol forme des dépressions qui se remplissent d'eau, se connectent, jusqu'à la formation d'un réseau d'écoulement de surface au cours de l'averse. Ce réseau conditionne l'efficacité du transfert et potentiellement, les échanges chimiques qui se produisent entre les eaux de ruissellement et la surface du sol. L'écoulement se fait ainsi sur des distances très variables, depuis la taille de la flaque, à quelques mètres, jusqu'au versant et au réseau hydrographique, selon les caractéristiques des pluies et de la surface. Un modèle numérique simulant le remplissage et la connexion des dépressions a été réalisé [15]. Par ce modèle, on montre qu'il existe un seuil de connexion de l'écoulement. Le coefficient de ruissellement passe d'une valeur proche de 0 à une valeur proche de $100 \%$ avec l'apport d'une faible quantité de pluie (figure 5). Ce seuil correspond à la détention superficielle. Paradoxalement, la quantité d'eau nécessaire pour remplir l'ensemble des dépressions est beaucoup plus forte que la détention superficielle. Ce modèle est utilisé pour examiner l'effet de la pente et de la taille du

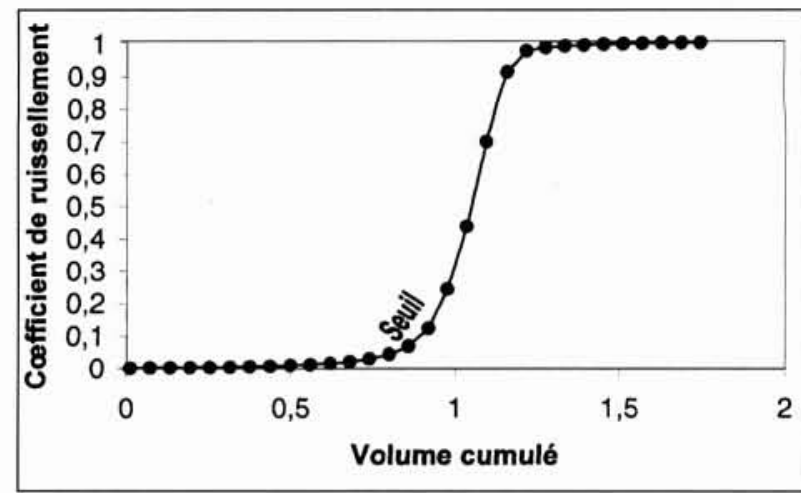

5. Evolution du coefficient de ruissellement en fonction de l'apport cumulé de pluie. Approche par modélisation numérique [15].

système sur la connexion de l'écoulement de surface. L'effet de la pente et de la taille du système sont déterminants sur l'apparition du seuil de déclenchement du ruissellement. Plus le système est de grande taille, plus le déclenchement et l'évolution de la distance de connexion des écoulements sont rapides. Ces approches sont importantes dans des crues moyennes où l'on voudra mieux évaluer les distances de transfert dans des milieux comportant différentes longueurs caractéristiques de rugosité, comme c'est le cas en milieu cultivé de topographie modérée.

L'ensemble de ces travaux sur le ruissellement confirme la variabilité et la multiplicité des facteurs contrôlant le ruissellement de surface, qui rend difficile sa quantification, même pour des crues moyennes. Dans les systèmes de grandes cultures et sous climat modéré, on avait essentiellement mis en avant un ruissellement hortonien, contrôlé par la dynamique très progressive d'évolution de la structure du sol, donnant un rôle privilégié à l'infiltrabilité des sols. On montre cependant que ce ruissellement ne peut pas être assimilé à une lame d'eau répartie de façon homogène, mais apparaît aussi contrôlé par la rugosité de la surface à différentes échelles : microrelief, motif agricole, pente, topographie qui conditionnent la propagation des écoulements selon des cheminement privilégiés et des seuils pluviométriques. Ces cheminements sont là aussi importants dans la mesure où ils vont conditionner l'acquisition de la qualité des eaux. Le contrôle hydrologique s'exerce également à d'autres échelles, en particulier dans le cas du ruissellement par saturation qui co-existe souvent lorsque les sols sont proches de la saturation. C'est le cas de sols où l'on trouve une semelle de labour, un imperméable ou une nappe peu profonde [16].

\section{V — LE RÔLE DE LA NAPPE À TRAVERS L'ÉTUDE DE SA DYNAMIQUE SPA- TIALE, SAISONNIÈRE ET ÉVÉNE- MENTIELLE}

Les travaux menés à la fois sur les zones contributives et sur le ruissellement ont montré l'importance qu'il y a à mieux connaître la configuration de la nappe et sa dynamique dans l'ensemble du bassin versant, pour mieux comprendre les processus de genèse des crues normales. Une approche expérimentale basée sur des observations hydrolo- 


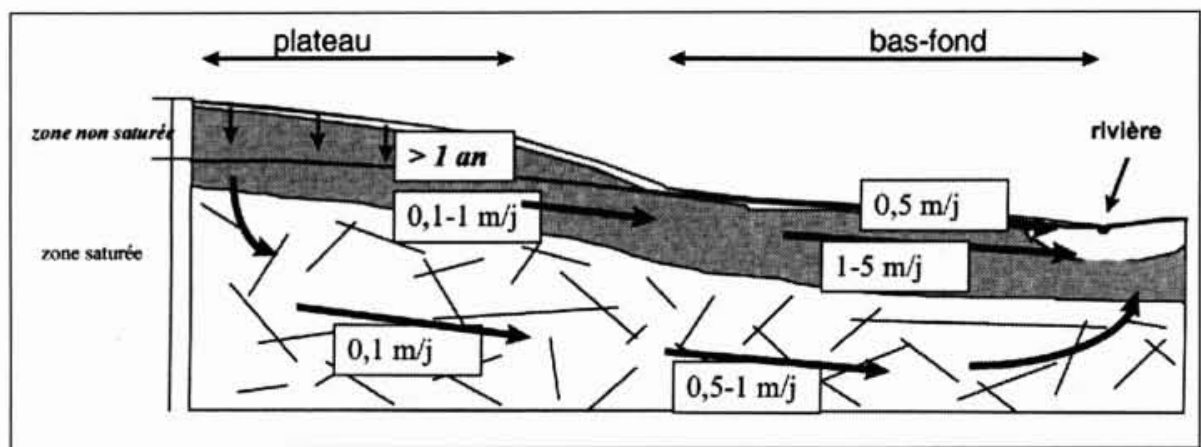

6. Configuration spatiale des différentes couches et de la nappe, vitesses de transfert associées [20].

giques a été menée sur le bassin versant du Coët-Dan à partir d'un suivi d'un dispositif de plusieurs piézomètres distribués selon la topographie. Deux périodes peuvent être distinguées pour décrire la dynamique de la nappe. En été et au début de l'automne la nappe n'est en général pas affectée par les pluies. En fin d'automne, pendant l'hiver et le printemps, la réponse de la nappe est rapide mais peu importante dans les zones de bas-fond où elle est proche de la surface. Sur les pentes et les plateaux, la nappe est située à une profondeur de l'ordre du mètre dans les versants, de quelques mètres sur les plateaux. Elle réagit alors aux fortes précipitations, avec parfois des fluctuations importantes et amorties. On a ainsi pu montrer la variabilité des gradients de nappe dans le temps et l'espace. Ces gradients jouent un rôle essentiel dans la contribution de la nappe à la rivière tant en crue et hors crue. Les gradients amont calculés dans le versant semblent jouer un rôle plus important que les gradients aval calculés dans la zone humide de bas-fonds. De plus, I'hypothèse souvent utilisée en modélisation hydrologique, de gradients hydrauliques parallèles à la surface topographique, limitée ici à quelques zones de bas fonds, apparaît ici remise en cause.

L'utilisation d'un modèle de nappe (MODFLOW), calé sur ces données piézométriques et paramétré par les caractéristiques physiques des différentes couches -sol, altérite de schiste et schistes, indique une contribution majeure de la nappe en période de crue, de $43 \%$ à $59 \%$ selon les événements pluvieux. La contribution de la nappe d'altérite y est dominante, à l'opposé celle de la nappe de schiste qui diminue fortement au cours de la crue, comme constaté par la méthode EMMA. Ces estimations sont cohérentes avec celles données par la méthode EMMA si l'on considère que la contribution de cette nappe est la somme des contributions issues de la zone humide et de la nappe, intégrant peut-être également pour partie la contribution du versant $[19,20]$. En intégrant à la fois les périodes de crue et hors crue, on estime la contribution globale de la nappe à environ $70 \%$. La nappe apparaît comme un réservoir hydrologique majeur, mobilisé très vite à la reprise hivernale des écoulements et lors de chaque crue en l'hiver. C'est un réservoir au sein duquel les transferts de l'eau sont relativement rapides avec des temps de résidence inférieurs à l'année dans le versant. A l'opposé sur les plateaux, ces transferts sont plus lents et impliquant des temps de résidence de l'eau pluriannuels (figure 6). L'origine des eaux en crue est ainsi de façon très prépondérante une eau ayant transité par les sols, les altérites et les substrats, une eau donc marquée par l'activité agricole, mais également conditionnée par les processus de transfert et de transformation de ces milieux.

\section{LE RÔLE DES RÉSEAUX LINÉAIRES : L'EXEMPLE DES RÉSEAUX DE HAIES}

L'influence des réseaux anthropiques sur les écoulements est de plus en plus souvent mise en avant en milieu rural. Nous évoquerons ici le rôle du réseau bocager sur les écoulements de crue. Son impact hydrologique a fait l'objet depuis peu d'une synthèse d'anciens travaux portant sur la comparaison de bassins versants bocager et ouvert [17]. C'est sur les crues normales que l'effet tampon du réseau bocager a pu être mis en évidence sur de petits bassins versants : ainsi, sur la fonction de transfert, on constate une réduction du pic de crue de 1,5 à 2 fois dans le bassin bocager ; sur la fonction de rendement, on observe que le coefficient de ruissellement est relativement constant dans le bassin bocager alors qu'il est plus variable (de 5 à $15 \%$ ) dans le bassin ouvert. Depuis, de nouveaux travaux ont été réalisés. On montre notamment que, dans un bassin versant bocager, le volume de crue dépend uniquement de la pluie totale, alors que sur un bassin versant ouvert, l'intensité de la crue et l'état d'humidité initial du bassin jouent également un rôle significatif (figure 7). Cela est cohérent avec les résultats obtenus en analysant les facteurs modifiés par la présence du bocage. L'extension de la zone saturée connexe au ruisseau est contrainte spatialement par les dispositifs talus-haie de ceinture de fond de vallée, et sa superficie est de ce fait relativement constante, alors qu'elle est beaucoup plus variable sur un bassin versant ouvert. Les facteurs sont à la fois d'ordre topographique et hydrologique, la dynamique saisonnière de la nappe étant localement modifiée du fait de l'évapotranspiration des arbres [17]. Par ailleurs, l'ensemble du réseau de haie conduit à déconnecter du réseau hydrographique une fraction de la surface du bassin génératrice des écoulements superficiels [20]. Cette fraction ne dépend pas de la densité totale du réseau bocager, mais de sa structure spatiale, c'est-à-dire de sa capacité à bloquer les écoulements de surface (figure 8).

\section{VII $\square$ CONCLUSION}

On a ici insisté, dans le cadre de l'analyse des crues normales, sur la contribution assez équilibrée des différentes composantes hydrologiques dans le contexte armoricain. On a présenté dans un premier temps les mécanismes hydrologiques liés au rôle des zones contributives de fond de vallée et au ruissellement de surface dans les versants, sur lesquels de nombreux travaux ont été réalisés ces vingt dernières années. On a ensuite montré l'importance d'une connaissance précise de la géométrie globale de la surface de la 


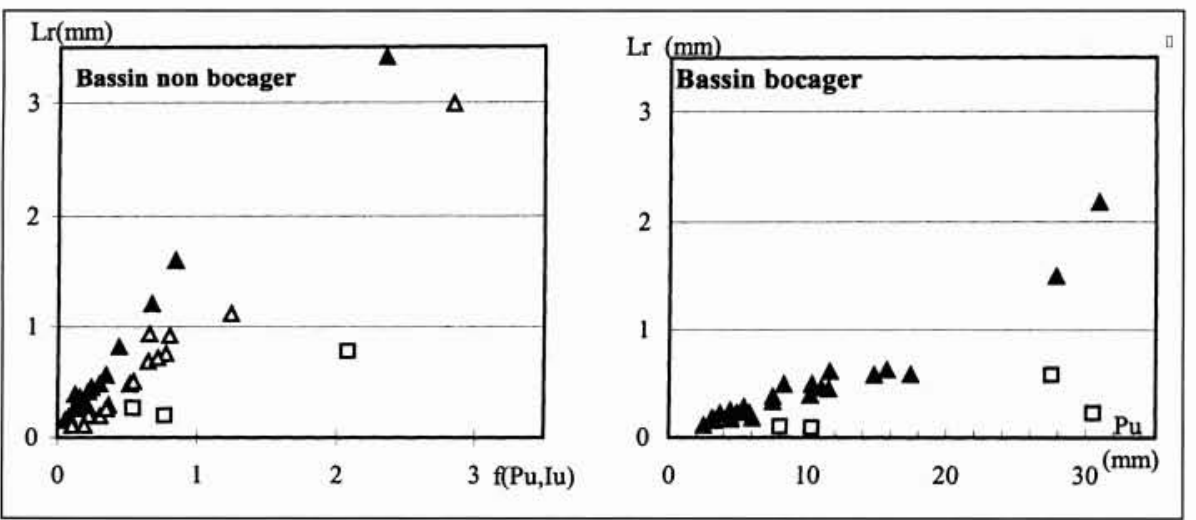

7. Relation entre les caractéristiques de la pluie utile et la lame ruisselée Ir. Les carrés représentent la période estivale, les losanges, la période hivernale.
Bassin bocager : relation entre la pluie utile et la lame ruisselée. Bassin non bocager : relation entre une fonction de la pluie utile et de son intensité,

(f $(\mathrm{Pu}, \mathrm{Iu})=\mathrm{Pu} 1,2$ Iu $0,6 / 47,6)$ et la lame ruisselée. (losange plein : crue groupée d'hiver; losange vide : crue isolée d'hiver; carré : crues d'été). On a distingué les crues isolées (triangles vides) et les crues groupées (triangles pleins). nappe dans les versants, des champs de vitesse associés, et enfin l'importance de la structuration des réseaux anthropiques sur les bassins versants, qui modulent l'amplitude de l'un ou l'autre des deux processus d'écoulement. Ces éléments permettent de préciser la géométrie réelle des zones contributives à l'écoulement sur le sol et à l'écoulement par les nappes.

\section{RÉFÉRENCES}

[1] MEROT P., BOURGET M. et LELEUCH M., 1981 Analyse d'une crue à l'aide du traçage naturel par l'oxygène 18 , mesuré dans les pluies, le sol, le ruisseau. CATENA, 8, 69-81.

[2] MEROT P., DURAND P , et MORISSON C., 1995. Four-component hydrograph separation using isotopic and chemical determinations in an agricultural catchment in western France. Phys Chem Earth. 20,3-4, 415425 .

[3] DURAND P. et JUAN TORRES J.L., 1996 - Solute transfer in agricultural catchments: the interest and limits of mixing models. J. Hydrol., 181, 1-22.

[4] CURMI P., DURAND P., GASCUEL-ODOUX C., HALLAIRE V., MEROT P., ROBIN P., TROLARD F., WALTER C. et BOURRIE G. 1995 - Le programme Cormoran-INRA : De l'importance des facteurs du milieu physique et de son aménagement dans le contrôle géochimique de la teneur en nitrates des eaux superficielles. Journal Européen d'Hydrologie, tome 26 , fasc. $1,37-56$.

[5] RIOU C., 1999. Géochimie des terres rares et des éléments traces associés dans les nappes et l'eau des sols hydromorphes. Application au traçage hydrologique. Thèse de l'Université de Rennes 1, 256 pp.

[6] CRAVE A. et GASCUEL-ODOUX C., 1997 - The influence of the topography on time and space distribution of soil surface water content. Hydrol. processes, 11, 203-210.

[7] WALTER C. et CURMI P., 1998. Les sols du bassin du Coët-Dan : organisation, variabilité spatiale et cartographie. Ed. C. Cheverry. Agriculture intensive et qualité des eaux., INRA éditions, 85-105.

[8] GASCUEL-ODOUX C, MEROT P., CRAVE A., GINESTE P., TAHA A. et ZHANG X., 1998. Les zones contributives de fond de vallée : localisation, structure, fonctionnement hydrodynamique. Ed. C. Cheverry. Agriculture intensive et qualité des eaux., INRA éditions, 129-142.

[9] BRUNEAU P., GASCUEL-ODOUX C., ROBIN P., MEROT P. et BEVEN K., 1995 - Sensitivity to space and time resolution of a hydrological model using digital elevation data. Hydrological Processes, Vol. 9 pp 69-81

[10] GINESTE P., 1998 - Contribution de l'imagerie satellitaire Radar ERS a la détection des zones saturées et à la modélisation hydrologique d'un petit bassin versant agricole par Top-Model. ENGREF.250 p

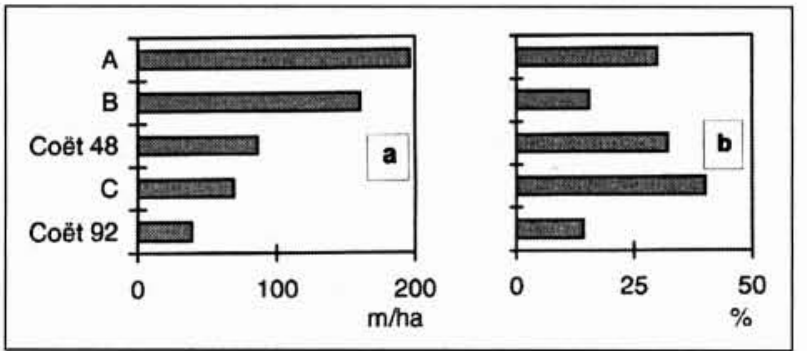

8. Influence d'un réseau de haies sur la connexion du réseau de drainage : a) densité du réseau de talus-haies sur 5 sites ; b) pourcentage de la superficie des bassins versants non connectée au réseau hydrographique.

11] GINESTE P., MEROT P. et PUECH C., 1997 - Dynamique du coefficient de rétrodiffusion du SAR d'ERS-1 en période ressuyage des sols d'un petit basin versant. Actes du septième symposium international ISPRS. Mesures physiques et signatures en télédétection. Courchevel, 711 avril 1997, Balkema éditeur. 7p.

[12] FRANKS S., GINESTE P., BEVEN K. et MEROT P., 1998 - On constraining the predictions of distributed models : the incorporation of fuzzy estimates of saturated areas into the calibration process, Water Resources Rechearch, 34, 4, 787 - 797

[13] GASCUEL-ODOUX, C., CROS - CAYOT S. et DURAND P., 1996. Spatial variations of sheet flow and sediment transport on an agricultural field. Earth Surface Processes and Landforms, vol.21, pp. 843-851

[14] HEDDADJ D. et GASCUEL-ODOUX C., 1999. Annual and topographic variations of hydraulic conductivity at the field scale. European J of Soil Sci., 50, 275-283.

[15] DARBOUX F., DAVY P., GASCUEL-ODOUX C. et HUANG C., sous presse. Evolution of soil surface roughness and flowpath connectivity in overland flow experiment. Catena.

[16] HUANG C.H., GASCUEL-ODOUX C., CROS-CAYOT S., sous presse. Hillslope moisture conditions, overland flow and erosion processes. Catena.

[17] MEROT P., sous presse. The Influence of Hedgerow Systems on the Hydrology of Agricultural Catchments in a Temperate Climate. Agronomie.

[18] MOLENAT J., DAVY P., GASCUEL-ODOUX C, et DURAND P., $1999^{-}$ Study of three subsurface hydrologic systems based on spectral and cospectral analysis of time series. Journal of Hydrology., 222 : 152-164

[19] MOLENAT J., 1999. Rôle de la nappe sur les transferts d'eau et de nitrates dans un bassin versant agricole. Etude expériementale et modélisation. Thèse Université de Rennes 1, 268 pp.

[20] MEROT P., GASCUEL-ODOUX C., WALTER C., ZHANG X. et MOLENAT J., 1999. Influence du réseau de haies des paysages bocagers sur le cheminement de l'eau de surface. Rev. Sci. Eau, 12/1, 23-44 